\begin{tabular}{|c|c|c|c|c|c|}
\hline Revista Clío América & ISSN: 1909-941X & Vol. 11 & No. 21 & julio - diciembre de 2017 & 169 - 176 \\
\hline \multicolumn{6}{c|}{ D0I: http://10.21676/23897848.2437 }
\end{tabular}

\title{
Competitividad, educación y empleabilidad: consideraciones para el desarrollo de los paises
}

Competitiveness, education and employability: considerations for the development of countries

\section{Jahir Enrique Lombana-Coy \\ Ph.D en Economía. Docente/Investigador, Universidad del Norte, Barranquilla, Colombia.Email: lombanaj@uninorte.edu.co ORCID: https://orcid. org/0000-0002-0055-8392}

\section{Alberto Elías Muñoz-Santiago}

Master en Economía Empresarial. Docente/ Investigador, Universidad del Norte, Barranquilla, Colombia.Email: amunoz@ uninorte.edu.co

ORCID: https://orcid. org/0000-0001-5921-0940

Tipología: Artículo de reflexión Fecha de recibido: abril 07 de 2017

Fecha de aceptación: noviembre 16 de 2017

Publicado en línea: noviembre 27 de 2017

Para citar este artículo: Lombana, C. J. y Muñoz, S. A. (2017). Competitividad, educación y empleabilidad: consideraciones para el desarrollo de los países. Clío América, 11(22), 169-176. Doi: $10.21676 / 23897848.2437$
RESUMEN: Las dinámicas socio-económicas de los países han resultado en el aumento de sus brechas de desarrollo. Existen aproximaciones al respecto que permiten rastrear las causas del desarrollo en el énfasis de factores productivos, particularmente entre los que prestan mayor o menor atención a una fuerza laboral formada para destrezas manuales 0 intelectuales. Este artículo busca hacer una reflexión sobre la relación entre competitividad, educación y empleabilidad para aportar a la discusión acerca de las brechas de desarrollo que se están acrecentando entre países. Basados en la revisión de autores que muestran la evolución de dicha relación, en cada sección se proponen reflexiones sobre el debate teórico, que dan pie a la constatación empírica particularmente para países en desarrollo.

Palabras clave: empleabilidad; competitividad; educación; desarrollo; competencias.

JEL: I25, M54

ABSTRACT: Specific socio-economic dynamics have resulted in increasing development gaps among countries. Some approaches attibute development to an emphasis on productive factors related to labor force formed for either manual skills, either intellectual ones. This article centers around the relationship between competitiveness, education and employability to contribute to this discussion. Based on the review of authors that show the evolution of this relationship between productive factors and development, each section proposes reflections on the theoretical debate and the empirical observation, particularly regarding developing countries.

Keywords: Employability; Competitiveness; Education; Development; Competences. 


\section{INTRODUCCIÓN}

El llamado paradigma de la globalización ha permeado una serie de ámbitos que traspasan el puramente económico. Procesos sociales, políticos, culturales, jurídicos entre otros han generado un cambio drástico en los procesos de educación, teniendo la innovación como una de sus variables dependientes y la tecnología como el vehículo que modifica los factores de producción de los economistas clásicos (tierra, capital y trabajo), y que precisamente resultan en un mercado laboral que cada vez debe ser más flexible y dinámico.

Gran parte de la explicación de las diferencias sustanciales existentes en el desarrollo de los países se debe a cómo han enfrentado el fenómeno de la globalización y cómo han aprovechado las ventajas de sus factores productivos. Se distinguen entonces dos grandes tendencias: economías con grados de desarrollo soportados en innovación y desarrollo de bienes de capital, sustentados en la ventaja competitiva (creación de valor); y economías emergentes y en desarrollo con modelos económicos soportados en bienes primarios y poca o ninguna cualificación de la mano de obra (valor heredado).

Este artículo busca, a través del análisis teórico y la revisión de la literatura, reflexionar sobre la relación que existe entre la competitividad, la educación y la empleabilidad como factores claves en el desarrollo de los países. Para lograr este objetivo, después de esta introducción se muestra brevemente la evolución de las teorías que buscan explicar la competitividad a través de la educación, cuestionando incluso la causalidad entre crecimiento y educación, que aún es motivo de debate. Al reconocer los diferenciales que pueden causar la (in)suficiente formación del capital humano en el desarrollo de los países, el artículo continúa con el debate teórico sobre las competencias requeridas por los potenciales trabajadores (capital humano) para poder ingresar al mercado laboral (empleabilidad). Luego se reflexiona sobre algunos alcances empíricos de la relación entre capital humano y empleabilidad. El artículo cierra con unas conclusiones.

\section{Competitividad y Educación: Evolución de Teorías}

La competitividad es un concepto elusivo y sus interpretaciones dependen de los niveles y las unidades de análisis en donde se utilice (Lombana, 2011). En las teorías clásicas de Adam Smith (2002) y David Ricardo (1993), la ventaja se basa en la especialización y el costo de oportunidad. Esto, con los factores tierra, capital y trabajo como constantes, lo que aduce implícitamente es que el cambio tecnológico y la innovación no serían tenidos en cuenta como fuentes de ventaja. Del análisis de la paradoja de Leontieff, que resulta del análisis de los autores Hecksher (1919) y Ohlin (1933), se da el avance de incluir el factor perdido de la tecnología como motor de ventaja, ya no únicamente comparativa como en los clásicos, sino competitiva en el sentido de la creación de valor. Es en el factor tecnológico donde se puede sustentar una definición de competitividad y donde el debate frente a la ventaja comparativa cobra relevancia. Mientras la ventaja comparativa se sustenta en el valor heredado, la ventaja competitiva se da en la creación de valor (Porter, 1990). El motor de la creación de valor es el cambio tecnológico (Schumpeter, 1934) propiciado por un conocimiento del ser humano para convertir y aumentar los factores de producción heredados (capital, tierra y trabajo) en productos creados.

La interpretación actual de los clásicos, particularmente de Adam Smith, propende por la no intervención del Estado. Adam Smith menciona en su Teoría de los Sentimientos Morales (2004) la obligación del Estado de ofrecer un marco jurídico, educación y cultura para garantizar el consenso moral de la sociedad, siendo de todas formas la educación parte del mercado. Mill (1951) y Marshall (2006) concuerdan con Smith en que el ser humano con el factor trabajo es generador de riqueza, aunque matizan la educación como parte del comportamiento de mercado (Oroval y Escardíbul, 1998).

Solamente con Theodor Shultz $(1960 ; 1963)$ en lo que denomina teoría del capital humano, y las aportaciones empíricas de Gary Becker (1983) y Jacob Mincer (1958), se ve al ser humano como generador de recursos que a partir de la educación 
pueden aumentar o mejorar la capacidad productiva de las personas. Esto se reflejaría según ellos en mejores salarios y en la posibilidad de incorporarse de manera más rápida al mercado laboral de donde se deriva el capital humano como complemento del capital físico. Estas concepciones soportaron las políticas públicas de los 70 al concebir el crecimiento económico basado fundamentalmente en la inversión en educación. Como crítica de la postura del capital humano está la postura de filtro de selección (Arrow, 1973; Spence, 1973; Stiglitz, 1973) que explica por qué las empresas contratan a las personas con mayor educación: les garantiza a las empresas menos gastos para su formación. También está la postura de Thurow (1972) en la competición por los puestos de trabajo, donde el trabajador es el que se adecúa a las necesidades de la empresa: el que lo haga más rápido es el que se queda 0 es apto para desempeñar una labor. Los que no entren en lo que llama Thurow "cola", pasan a ser desempleados por no cumplir con los requisitos de los empleadores.

Las posturas de Doeringer y Piore (1971) se centran en la concepción de un mercado interno en el que la formación de los individuos hace que el empleador propenda por mantener a sus empleados y que la formación primordial sea dada por la empresa y no por el sistema educativo formal. Esto hace que el mercado al que llaman interno sea altamente cualificado, especializado y a la vez rígido. En un trabajo posterior, Piore (1983) agrega a este mercado interno, primario, un mercado externo en donde están los trabajadores que no han alcanzado un grado de especialización, tienen salarios bajos y alta inestabilidad laboral, constituyéndose en otra postura crítica del capital humano. Finalmente, existe una visión radical (Bowles y Gintis, 1976) que presenta al sistema educativo moderno como un reflejo de la desigualdad en las empresas, con gerentes bien capacitados que dominan una fuerza laboral poco educada y a la cual el sistema no permite nuevas opciones a menos de que esté en la parte alta de las relaciones de producción y propiedad.

Las perspectivas del capital humano y sus críticas coinciden en que finalmente el factor tecnológico, ya sea por educación o innovación, es el que podría definir la competitividad (Lombana, 2012); sin embargo, la evidencia empírica sobre su causalidad aún no es concluyente.

Un problema que se presenta está en el respaldo empírico de causalidad entre crecimiento y educación (Arellano, 2002). Por una parte, Solow (1956) explica que el crecimiento del PIB está representado por una ecuación cuyas variables dependientes son los factores de producción; sin embargo, el residuo estaría representando de acuerdo a este análisis del conocimiento resultante del capital humano (World Bank, 1999). Otros trabajos (Lucas, 1988; Romer, 1989; Krugman, 1993 y OCDE, 1996) confirman la relación positiva entre educación y crecimiento. Por otra parte, (Krueger y Lindhal, 2000; Bellei y González, 2003; Duncan, 2010) reconocen la relación, pero aducen que la variable representada en el residuo, para varios enfoques en cobertura, calidad, distribución de gastos para diferentes niveles de educación (por ejemplo, primaria, secundaria y terciaria) podrían no solo no causar efecto en el crecimiento, sino incluso afectarlo negativamente.

Una ruptura en la concepción utilitarista del capital humano y en las vertientes críticas anteriormente mencionadas, se da con la incorporación del factor tecnológico como explicación del crecimiento. Así, no es solo la educación del capital humano la que explica el crecimiento, sino la creación de valor por los conocimientos adquiridos durante la vida de los individuos reproducidos en innovaciones ante "situaciones de mercado y no mercado" (OCDE, 1996, p.22). En este nuevo contexto, la creación de valor y la innovación constituyen el elemento a consensuar en una definición de competitividad.

Gran parte de las brechas presentadas entre países desarrollados y en desarrollo se sustenta en el hecho de que los últimos han soportado su desarrollo en aprovechamientos de las ventajas comparativas (producción de bienes primarios), incluyendo desarrollos mínimos o nulos basados en la innovación, por lo que la mano de obra se ha concentrado en realizar labores con bajo contenido en conocimientos y fuertemente apalancadas en destrezas físicas. Mientras tanto, los otros han utilizado sus escaseces para fortalecer la innovación y el desarrollo de tecnologías generadoras de valor agregado, con el consiguiente fortalecimiento cualitativo de la mano 
de obra. De esto se desprende que difícilmente se podrá utilizar tecnología de punta si no se cuenta con capital humano capacitado para entenderla, utilizarla y sacar el máximo provecho en términos de eficiencia y productividad, lo que redunda en menos posibilidades de empleabilidad que es sobre lo que se reflexiona en la siguiente sección.

\section{Capital Humano Hacia la Empleabilidad}

Los individuos pueden tener potencial para realizar determinada actividad, por ello, además de las competencias adquiridas por la formación, deben tener habilidades y capacidades pertinentes para desempeñar determinada labor. Cuando ese potencial individual se encuentra con la necesidad de una empresa, se habla del concepto de empleabilidad.

Aunque existen matices sobre la definición de empleabilidad (Hillage y Pollard, 1982; Harvey, 2001; Barrie, 2004; Weinberg, 2004; McQuaid y Lindsay, 2005; Cala, Gutiérrez, Barragán y Valero, 2011; Formichella y London, 2013 y Lantarón, 2014) ya sea desde el punto de vista del grupo de interés (empleadores, Instituciones de Educación Superior, gobierno, estudiantes) o ya sea desde la disciplina (ingenierías, ciencias básicas, administración, etc.), todas las definiciones se atan a factores de formación (adquirida y requerida), inserción profesional, permanencia y sostenibilidad laboral y adquisición de competencias. La diferenciación de los individuos en alguno de estos factores es lo que permite a las empresas seleccionar personal de un conjunto de aspirantes altamente competentes.

El estudio de las competencias nace en el campo laboral, por lo tanto, es pertinente preguntarse cuáles son las más importantes para un buen desempeño en el trabajo. Dentro de las teorías de los mercados laborales, la corriente de pensamiento que prepondera las características de la fuerza de trabajo como unidades de análisis, revela que el conocimiento, como inversión, incrementa la productividad y es sustento de la llamada teoría del capital humano (Schultz, 1983; Becker, 1983; Mincer, 1981). Campos-Ríos (2003) explica que es en esta teoría donde el concepto de empleabilidad cobra importancia, principalmente cuando, en la práctica, la oferta laboral excedente genera brechas que no son cubiertas por el mercado y que aquellos que tienen el conocimiento acceden de manera preferencial a las plazas ofrecidas. Sin embargo, dicho conocimiento no es solamente el adquirido de manera formal sino también por la experiencia u otras fuentes, que en su conjunto forman el acervo de competencias de los individuos requeridos para la productividad, lo que se ha denominado capital humano (OCDE, 2007).

Es en el siglo XIX con Max Weber (1924) cuando se rastrea la aplicación de encuestas para la selección de personal y se verifica la voluntad o el deseo de un individuo de realizar un trabajo. Es con Becker (1983) en el siglo XX cuando cobra importancia la racionalidad económica en la decisión de emplearse, esto es que con mayor conocimiento hay más oportunidades de acceder al mercado laboral. A pesar de que en la teoría se menciona el capital humano, este no necesariamente se vincula al concepto de empleabilidad, sino solamente y de manera empírica con Piore y Doeringer (1985), quienes representan la situación física de un potencial empleado, aspirando a un puesto de trabajo y "haciendo la fila" para llegar primero y acceder a él. Así, los que están primeros en la fila tienen el acervo de conocimientos, capacidades y habilidades para acceder a los mejores trabajos, mientras que los menos capacitados acceden a trabajos marginales o no acceden. De este símil de la fila se diferencian las posiciones de Arrow (1972) y Thurow y Lucas (1972) en que, aunque el conocimiento sí puede ser un medio para acceder a un empleo, no garantiza una productividad mayor en el puesto de trabajo.

Dicho lo anterior, en un escenario de sobreoferta, los empleadores tienen un amplio abanico de posibilidades para escoger a sus empleados, pero el dilema surge en encontrar el empleado adecuado de acuerdo a los intereses de la empresa. Wentling (1987) menciona que el problema no está en las habilidades técnicas (específicas) de los graduados, sino en las habilidades no técnicas (genéricas). El trabajo empírico de Beach (1982) corrobora que las competencias específicas (en el primer empleo) son menos importantes que las genéricas. Adicionalmente, el trabajo de Cotton (1993) ponderó en un cuestionario enviado a empresarios que las capacidades básicas primaban en la decisión de contratación sobre las específicas. 
Existen tres corrientes contrarias (Gil-Villa, 1995) a la teoría del capital humano en su carácter de motivador de la productividad: 1) la creación de tecnologías de la información y las comunicaciones no necesariamente mejoran los puestos de trabajo, de hecho se aduce que los puestos operativos menos calificados son los que más crecen (Levin y Rumberger, 1986); 2) la sobre-educación o la idea de que la escolarización de los individuos avanza más rápido que los puestos y que hay casos donde el empleado considera que no habría tenido necesidad de estudiar para realizar sus actividades (Zárraga 1985); y 3) la concepción de Thurow: el puesto de trabajo es el que da las competencias y no la formación previa.

Tanto en detractores como seguidores de la teoría del capital humano existe consenso en que el conocimiento, de una u otra forma, está vinculado al desarrollo económico, ya sea utilizando como vehículo la productividad, como en el caso de los seguidores, o como uno más de los elementos a analizar, en los detractores. Cuando se vincula este debate al concepto de empleabilidad, las Instituciones de Educación Superior (IES) juegan un papel fundamental, pues se discute la coherencia entre las necesidades del mercado laboral y la oferta académica que las IES están dando a sus estudiantes para cerrar las brechas entre ambos. Lantarón (2014) presenta este debate en las universidades orientándose por un lado a los planes de estudio y por otro a la asesoría directa a los estudiantes y graduados, en lo que encuentra coincidencia con varios autores (Ayast, 2010; Harvey, 2001; Kohler, 2004; Mora, 2011; Knight y Yorke, 2004; Pastor, Simón, García y Tovar, 2004 y Teichler 2004, 2008), quienes referencian la empleabilidad como un tema requerido en las IES. En cuanto a los planes de estudio, hay consenso en que las IES deben apoyarse en otros grupos de interés (empleadores, asociaciones empresariales, gobierno) (Ortoll, 2008; European Commission, 2010; Rodríguez, Prades, Bernáldez y Sánchez, 2010; Cabeza, Castrillón y Lombana, 2012; Speight, Lackovic y Cooker, 2013; Cai, 2013; Tymon, 2013) y en que las competencias a desarrollar deben dividirse en genéricas y específicas para el área disciplinar (Lombana, Cabeza, Castrillón y Zapata, 2014). En cuanto al apoyo en oficinas especia- lizadas para la empleabilidad, los estudios se limitan a la revisión no exhaustiva de las oficinas de graduados en universidades (Cardoso, Escária, Ferreira, Madruga, Raimundo y Varanda, 2014) y de las oficinas gubernamentales nacionales y locales dedicadas a esta labor (ver por ejemplo la aproximación institucional de Cai, 2013).

\section{Empleabilidad en la Práctica}

Las realidades de empleabilidad difieren por regiones, no en vano el Foro Económico Mundial (2014) la coloca entre sus mayores preocupaciones: "muchos graduados recientes están descubriendo que a pesar de sus calificaciones académicas -a menudo a expensas significativas- carecen de las habilidades técnicas y profesionales específicas que demanda el siempre cambiante mercado laboral" (p. 36).

En los países de la Organización para la Cooperación y el Desarrollo Económicos existe la preocupación del desplazamiento de las empresas a países con mano de obra más barata, muy a pesar de la calidad educativa en los países desarrollados, que no resiste la competencia de costos laborales bajos de los países en vía de desarrollo. Solamente en los trabajos de base en el conocimiento para nuevas tecnologías (en su mayoría países desarrollados) han tenido aciertos en la vinculación laboral, pero la migración de expatriados puede repercutir de manera perversa en la empleabilidad de los países desarrollados. Finalmente, está el incremento de egresados de la educación superior que empieza a exceder la capacidad de absorción del mercado laboral y que también se observa en otros países de la OCDE (Pavlin y Svetlik, 2014). Estas disparidades entre políticas y resultados ponen a países desarrollados a repensar la coherencia entre el mercado laboral y las decisiones de política (en el caso de Europa, de la declaración de Bologna) y, como menciona Leoni (2014), queda el cuestionamiento de si se remunera por la educación formal, por las competencias o por la combinación de ellas.

Si bien cada día se gana conciencia de lo importante de sustentar toda la política gubernamental en una sólida, realista y viable política educativa, se observan fenómenos como sobre oferta de mano de obra en muchas áreas, escases en algunas donde 
se requeriría, dada la posición estratégica para el desarrollo económico de los países, y subempleo que puede incluso subvalorar las estadísticas oficiales.

\section{CONCLUSIÓN}

En el mundo se observa que las diferencias entre países desarrollados y en vía de desarrollo son cada día más amplias, fundamentalmente por incrementos en los niveles de competitividad en los primeros, producto de una política económica basada en generación de valor.

Una economía sustentada en principios de generación de valor da importancia enorme a la innovación, generando un efecto multiplicador para nuevo conocimiento $y$, por lo tanto, modificando la composición en cantidad y calidad de la oferta y la demanda laboral.

Esto hace evidente que existe una relación directa entre competitividad, educación y empleabilidad. Sin embargo, no hay un consenso entre la causalidad, particularmente entre competitividad y educación. Lo que sí es claro es que de la educación, ya sea formal o informal, dependerá el grado de absorción laboral del aparato productivo que, especialmente en países en desarrollo, ha generado impactos negativos por sustentar la economía en producción y exportación de bienes primarios 0 commodities, provocando un estancamiento en la formación de mano de obra, dirigiéndola sobre todo a la generación de destrezas manuales, más que a destrezas intelectuales. Para países en desarrollo, la revisión y la reestructuración del sistema educativo es una necesidad prioritaria, estableciendo políticas de largo plazo que fomenten el desarrollo de nuevo conocimiento y no la repetición de teorías en muchos casos obsoletas en el mundo.

Queda en el debate si se deben priorizar competencias genéricas o específicas en la formación, pero, de acuerdo a los ejercicios empíricos, las genéricas son el primer elemento a tomar en cuenta por los empleadores para contratar; lo específico se supone aprendido por las credenciales presentadas 0 se aprende en el puesto de trabajo. Al ver vacíos empíricos y debates sin concluir, en países en desarrollo y en la disciplina administrativa se requiere hacer estudios empíricos de las percepciones de diferentes grupos de interés sobre las competencias requeridas para la empleabilidad, que abonen el terreno para cerrar brechas de competitividad.

\section{REFERENCIAS BIBLIOGRÁFICAS}

Arellano, J. P. (2002). Competitividad internacional y educación en los países de América Latina y el Caribe. Revista Iberoamericana de Educación, (30), 63-104.

Arrow, K. (1972). Higher education as a filter. Technological report. Standford: Stanford University.

Arrow, K. (1973). Higher education as a filter. Journal of Public Economics, 2(3), 193-216.

Ayast, J.C. (2010). Estrategias de la Universidad Politécnica de Valencia para la empleabilidad de sus titulados en el marco de las relaciones UniversidadEmpresa. Tesis doctoral. Valencia: Universidad Politécnica de Valencia.

Barrie, S. (2004). A research-based approach to generic graduate attributes policy. Higher Education Research and Development, 23, 261-275.

Beach, D. (1982). A training program to improve work habits, attitudes, and values. Journal of Epsilon Pi Tau, 8(2), 69-74.

Becker, G. S. (1983). El capital Humano. Alianza Universidad de Textos, Madrid: Alianza Editorial, S.A.

Bellei, C. y González P. (2003). Educación y competitividad en Chile. Documento de trabajo. Santiago: Educar Chile. Recuperado de http://ww2.educarchile. cl/UserFiles/P0001/File/Educ\%20Comp\%20en \%20 chile.pdf

Bowles, S. y Gintis H. (1976) Schooling in capitalist America: Educational reform and the contradictions of economic life. Abingdon: Routledge \& Kegan Paul ed.

Cabeza, L., Castrillón, J. y Lombana, J. (2012). Determinación y análisis de las competencias por fortalecer para la gestión en Colombia. Innovar, 22(46), 5-20.

Cai, Y. (2013). Graduate employability: a conceptual framework for understanding employers' perceptions. Higher Education, 65, 457-469.

Cala, M., Gutiérrez, C., Barragán, C. y Valero, P. (2011). Empleabilidad: una aproximación a la conceptualización. Cuadernos Hispanoamericanos de Psicología, 11(1), 7-18.

Campos-Ríos, G. (2003). Implicaciones del concepto de empleabilidad en la reforma educativa. Aportes, (23), 101-111. Benemérita Universidad Autónoma de 
Puebla, México Recuperado de http://www.redalyc. org/pdf/376/37602308.pdf

Cardoso, J., Escária, V., Ferreira, V., Madruga, P., Raimundo, A. y Varanda, M. (2014). Employability and higher education in Portugal. Journal of Graduate Employability, 0, 17- 31.

Cotton, K. (1993). Developing Employability Skills. Close Up \#15. Portland: School Improvement Research Series. (SIRS)

Doeringer, P. y Piore, M. (1971). Internal Labor Markets and Manpower Analysis. Lexington, Mass: Harvard University.

Duncan, A. (2010). Back to School: Enhancing U.S. Education and Competitiveness. Foreign Affairs, 89(6), 65-76.

European Commission - EC. (2010). Employers' perception of graduate employability. Analytical report. Flash Eurobarometer 304 - November. Brussells: The Gallup Organization.

Formichella, M. y London, S. (2013). Empleabilidad, educación y equidad social. Revista de Estudios Sociales, 47, 79-91.

Foro Económico Mundial. (2014). Global Risks Ninth Edition. Davos: WEF. Recuperado de http://www3. weforum.org/docs/WEF_GlobalRisks_Report_2014. pdf

Gil-Villa, F. (1995). El estudiante como actor racional: objeciones a la teoría del capital humano. Revista de Educación, 306, 315-327

Harvey, L. (2001). Defining and measuring employability. Quality in Higher Education, 7(2), 97- 109.

Heckscher, E. (1919). The Effect of Foreign Trade on the Distribution of Income. Ekonomisk Tidskrift, 497-512.

Hillage, J. y Pollard, E. (1982). Employability: Developing a framework for policy analysis. London: Department for Education and Employment.

Knight, P. y Yorke, M. (2004). Learning, curriculum and employability in Higher Education. London: Routledge Falmer.

Kohler, J. (2004). The Bologna Process and employability: The impact of employability on curricular development. Seminario La empleabilidad y su vinculación con los objetivos del Proceso de Bolonia, celebrado en Slovenia en octubre de 2004. Recuperado de http://www.aic.Iv/ bolona/Bolgna/Bol_semin/Bled/041022_Kohlersp.pdf

Krueger, A. y Lindhal, M. (2000). Education for Growth: Why and for Whom? NBER Working Paper Series. Working Paper 7591. Recuperado de http://www. nber.org/papers/w7591
Krugman, P. (1993). Toward a Counter-Counterrevolution in Development Theory. Proceedings of the World Bank Annual Conference on Development Economics Washington, D.C.: World Bank.

Lantarón, B. (2014). La empleabilidad en la Universidad Española. Journal for Educators, Teachers and Trainers, 5(2), 272-286.

Leoni, R. (2014). Graduate employability and the development of competencies. The incomplete reform of the "Bologna Process", International Journal of Manpower, 35(4), 448-469.

Levin, H. y Rumberger, R. (1988). Las nuevas tecnologías y las necesidades educativas: visiones, posibilidades y realidades. Revista de la Universidad Complutense Política y Sociedad, 1, 7-21.

Lombana, J. (2011). Looking for a Distinctive Model with which to Analyze Competitiveness. Advances in Competitiveness Research; 19(3/4), 32-44.

Lombana, J. (2012). Pertinencia de la Educación en la Competitividad. Revista Zona Próxima, 16, 69-85.

Lombana, J., Cabeza, L., Castrillón, J. y Zapata, A. (2014). Formación en competencias gerenciales. Una mirada desde los fundamentos filosóficos de la administración. Estudios Gerenciales, 30(132), 301-313.

Lucas, R. E. (1988). On the mechanics of economic development. Journal of Monetary Economics, 22, 3-22.

Marshall, A. (2006) Principios de Economía. Editorial Síntesis: Madrid, España.

McQuaid, R. y Lindsay, C. (2005). The concept of employability. Urban Studies, 42(2), 197-219.

Mill, J. (1951). Principios de economía política: con algunas de sus aplicaciones a la filosofía social. Fondo de Cultura Económica: Buenos Aires, Argentina.

Mincer, J. (1958). Investment in human capital and personal income distribution. Journal of Political Economy, 66(4), 281-302.

Mincer, J. (1981). Human Capital and Economic Growth. NBER: Working Paper Series. Working Paper No. 803. National Bureau of Economic Research. Recuperado de http://www.nber.org/papers/w0803.pdf

Mora, J. (2011). Las competencias relacionadas con la empleabilidad. Ponencia presentada en el Seminario Bienal de Educación Superior y Empleabilidad, organizado por la Cátedra Unesco de Gestión y Política Universitaria en Madrid en 2011.

Organización para la Cooperación y el Desarrollo Económico - OCDE. (2007). Insights Human Capital: How What You Know Shapes Your Life. 
Paris: OCDE. Recuperado de https://www.oecd.org/ insights/38435951.pdf

Ohlin, B. (1933). Interregional and International Trade. Cambridge: Harvard University Press.

Organización para la Cooperación y el Desarrollo Económico - OCDE. (1996). Measuring what people know: Human capital for the knowledge economy. Paris: OCDE.

Oroval, E. y Escardíbul J. (1998). Economía de la Educación. Madrid: Ediciones Encuentro.

Ortoll, E. (Ed.) (2008). Análisis de los procedimientos de interacción entorno-universidad en el proceso de adaptación e implementación de las titulaciones oficiales del EEES. Madrid: MEC-Programa de estudios y análisis.

Pastor, M.S., Simón, L., García, L. y Tovar, E. (2004). Las demandas sociales y su influencia en la planificación de las titulaciones en España en el marco del proceso de convergencia europea de educación superior. Madrid: MEC-Programa de estudios y análisis.

Pavlin, S. y Svetlik, I. (2014). Employability of higher education graduates in Europe. International Journal of Manpower, 35(4), 418-424.

Piore, M. y Doeringer, P. (1985). Mercados Internos de Trabajo y Análisis Laboral (2a edición). Madrid: Ministerio de Trabajo y Seguridad Social. España.

Piore, M. (1983). Labor Market Segmentation: To What Paradigm Does It Belong? American Economic Review, 73(2), 249-53.

Porter, M. (1990). La ventaja competitiva de las naciones. Buenos Aires: Editorial Vergara.

Ricardo, D. (1993. Principios de Economía Política y Tributación. Bogotá: FCE.

Rodríguez, S., Prades, A., Bernáldez, L. y Sánchez, S. (2010). Sobre la empleabilidad de los graduados universitarios en Catalunya: del diagnóstico a la acción. Revista de Educación, (351), 107-137

Romer, P. (1989). Human Capital and Growth Theory Evidence, NBER Working Paper Series, núm. 3173, National Bureau of Economic Research, Inc., Cambridge, Ma.

Schultz, W. (1983). La inversión en capital humano. Educación y Sociedad, 1, 180-195.

Schumpeter, J. (1934) The Theory of Economic Development. Cambridge: Simpson.

Shultz, T. (1960). Capital formation by Education. Journal of Political Economy, 68, 571.
Shultz, T. (1963). The Economic Value of Education. New York: Columbia University Press.

Stiglitz, J. (1973). The theory of "screening" education and the distribution of income Cowles Foundation Discussion Paper 354.

Smith, A. (2002). La riqueza de las naciones. Madrid: Alianza Editorial.

Smith, A. (2004). Teoría de los sentimientos morales. Madrid: Alianza editorial.

Solow, R. (1956). A Contribution to the Theory of Economic Growth. Quarterly Journal of Economics, 70(1), 65-94.

Speight, S., Lackovic N. y Cooker, L. (2013). The Contested Curriculum: Academic learning and employability in higher education. Tertiary Education and Management, 19(2), 112-126.

Spence, M. (1973). Job Market Signaling. The Quarterly Journal of Economics, 87(3), 355-374.

Teichler, U. (2004). Changes in the relationships between Higher Education and the world of work on the way towards the EHEA. EUA Conference University and Society Engaging Stakeholders. Marseille, April 2004.

Teichler, U. (2008). Employability and the curriculum. Taller sobre empleabilidad en la formación universitaria, organizado por la Dirección General de Universidades y la Universidad de León. Enero de 2008.

Thurow, L. y Lucas, R. (1972). The American Distribution of Income: A Structural Problem. Washington: U.S. Congress.

Thurow, L. (1972) The Economics of Overeducation. Economics of Education Review, 4, 93-104.

Tymon, A. (2013). The student perspective on employability. Studies in Higher Education, 38(6), 841-856.

Weber, M. (1924) Sociología del trabajo industrial. Madrid: Ed. Trotta.

Weinberg, P. (2004). Formación profesional, empleo y empleabilidad. Porto Alegre: Foro Mundial CINTEFOR

Wentling, R. (1987). Employability Skills: The Role of Business Education. Journal of Education for Business, 62(7), 313-317.

World Bank. (1999). World Development Report: Knowledge for Development 1998/1999. Washington D.C.: World Bank.

Zárraga, J. (1985). Informe: Juventud en España. Madrid: Ministerio de Cultura. 